\title{
Chemically Interconnected Light-Weight 3D-Carbon Nanotube Solid Network
}

Sehmus Ozden ${ }^{1}$, Thierry Tsafack ${ }^{2}$, Peter S. Owuor ${ }^{2}$, Yilun Li $^{3}$, Almaz S. Jalilov ${ }^{4}$, Robert Vajtai ${ }^{2}$, Chandra S. Tiwary', Jun Lou ${ }^{2}$, James M. Tour ${ }^{3}$, Aditya D. Mohite ${ }^{1}$, Pulickel M. Ajayan ${ }^{2 *}$

${ }^{1}$ Materials Physics and Applications Division, Los Alamos National Laboratory, Los Alamos NM, 87545, USA

${ }^{2}$ Department of Material Science and NanoEngineering, Rice University, Houston, Texas 77005, USA

${ }^{3}$ Department of Chemistry, Rice University, Houston, Texas 77005, USA

${ }^{4}$ Department of Chemistry, King Fahd University of Petroleum and Minerals, Dhahran 31261, Saudi Arabia

KEYWORDS: Three-Dimensional nanotubes, $\mathrm{CO}_{2}$ uptake, fracture mechanism, chemical crosslinking, MD simulations

\section{ABSTRACT}

Owing to the weak physical interactions such as van der Waals and $\pi-\pi$ interactions, which holds nanotubes together in carbon nanotube (CNT) bulk structures, the tubes can easily slide on each other. Creating covalent interconnection between individual carbon nanotube (CNT) structures could remarkably improve the properties of their three-dimensional (3D) bulk structures. The creation of such nanoengineered 3D solid structures with improved properties and low-density remains one of the fundamental challenges in real-world applications. Here, we report the scalable synthesis of low-density 3D macroscopic structure made of covalently interconnected nanotubes using free-radical polymerization method after functionalized CNTs with allylamine monomers. The resulted interconnected highly porous solid structure exhibits higher mechanical properties, larger surface area and greater porosity than non-crosslinked nanotube structures. To gain further insights into the deformation mechanisms of nanotubes, fully atomistic reactive molecular dynamics simulations are used. Here we demonstrate one such utility in $\mathrm{CO}_{2}$ uptake, whose interconnected solid structure performed better than non-interconnected structures.

\section{INTRODUCTION}


The fabrication of macroscopic three-dimensional (3D) solid structures of nanotubes from chemical interconnection of their individuals is a promising approach to harness the outstanding properties of nanotubes for development of new engineering materials [1-5]. Individual nanotubes in the as grown bundle stick together via weak physical van der Waals, $\pi-\pi$ and hydrophobic interactions thereby making it easier to collapse in the presence of load thus limiting their physical properties as well as their performances in applications. Crosslinking individual nanotubes is one of the desirable methods to improve mechanical and physical properties of bulk structures [6,7]. Additionally, generating intermolecular junction between individual nanotubes exhibits interesting morphological and unique properties [4,8]. By controlling atomic-scale bridging between individual nanotubes, they can be ordered as covalently bonded 3D-solid networks with controllable physical properties such as density, porosity and mechanical properties $[9,10]$.

Even though several chemical vapor deposition (CVD) methods [11, 12], surface chemistry methods $[13,14]$, and welding techniques $[15,7]$ have been reported for building such 3D structures of nanotubes by creating atomic-scale bridges between individual tubular structures of CNTs, the fabrication of such nanoengineered 3D architectures remains one of the most significant challenges to integrate these materials in practical applications. Among all these approaches, the most promising and controllable approach is surface chemistry method because the properties of nanostructures can easily be manipulated by playing on surface functionalities. Additionally, this approach is crucial for the inexpensive mass production and selectivity of 3DCNT solid network structures. CNT materials were produced using chemical functionalization, consisting of bonds with amines functional group. Recently, we reported Suzuki cross-coupling reaction for interconnection of individual CNTs using conjugate molecules to create light-weight and porous solid building blocks [13]. Such porous solid materials have a number of interesting properties such as lightweight, high-surface area etc. Their unique structure and exceptional properties demonstrated potential broad range of applications ranging from gas adsorption [14] [14], energy storage [16], environmental application [13] and mechanical damping [6]. For example, A. D. Leonard and co-workers used diazonium coupling chemistry for crosslinking carbon nanotubes [14]. As a result of diazonium coupling reaction, they obtained 3D-CNTs structures and used effectively for hydrogen storage [14]. In the other example, J. Zhu and coworker used carboxylic acid functionalized single-walled CNTs and diamine terminal epoxy 
polymer for covalently interconnecting CNTs [17]. In this report, we functionalized surface of CNTs with allylamine monomer and used free-radical polymerization to create 3D-CNT solid structure (Figure 1) and used the resulted light-weight porous architecture for $\mathrm{CO}_{2}$ adsorption.

\section{EXPERIMENTAL SECTION}

Multiwall carbon nanotubes (outer diameter:20-30nm, inner diameter:5-10nm, length:1030 $\mathrm{m}$, Purity: >95 wt\%) obtained from cheaptubes.com. The obtained CNTs were stirred in concentrated $\mathrm{HNO}_{3}$ for 6 hours at $70^{\circ} \mathrm{C}$ to produce shortened $\mathrm{CNTs}$ and purify nanotubes from unwanted amorphous carbon and residual metal catalyst impurities. Additionally, the oxidation process produces oxygen containing functional groups, mainly carboxyl groups. The oxidized nanotubes filtered and washed with DI-water until pH:7 and dried overnight at $60{ }^{\circ} \mathrm{C}$. . After the oxidation process, the carboxyl groups were converted to acid chloride by reacting with thionyl chloride $\left(\mathrm{SOCl}_{2}\right)$ at $80{ }^{\circ} \mathrm{C}$ for $24 \mathrm{~h}$ and the resulted material filtered and washed with anhydrous ether. Then, CNTs were functionalized with allylamine monomer In detailed, CNTs $(0.5 \mathrm{~g})$ dispersed in 50ml DMF for 2 hours sonication and allylamine monomer added into the dispersed CNTs solution with $5 \mathrm{~mL}$ trimethylamine. The mixture was stirred at $25{ }^{\circ} \mathrm{C}$ for $24 \mathrm{~h}$ under nitrogen, then washed with excess DI-water to remove unreacted allylamine.3D-CNT solid structures was produced by free radical polymerization of nanotube monomers in DMF using azobisisobutyronitrile, AIBN (1 mol\%) as initiator [18]. The polymerization reaction was performed at $80 \circ \mathrm{C}$ for $24 \mathrm{~h}$ under nitrogen atmosphere. The final crosslinked nanotubes filtered and washed with DI-water. To create 3D solid structures, crosslinked nanotubes sonicated and freeze in liquid nitrogen and 3D porous CNTs solids obtained after freeze-drying process. The reaction was comfirmed by Raman spectroscopy, Fourier transform infrared spectroscopy (FTIR), X-ray photoelectron spectroscopy (XPS), scanning electron microscope (SEM, FEI Quanta 400 ESEM FEG), and transmission electron microscope (JEOL 2100 Field Emission Gun TEM). Quantachrome BET surface analyzer was used to determine the surface area and pore size distribution. Here we also demonstrate the potential of the $3 \mathrm{D}$ solid macrostructure for $\mathrm{CO}_{2}$ storage.

\section{RESULTS AND DISCUSSION}

The crosslinking mechanism between nanotubes rely on free-radical polymerization of allylamine monomer that covalently attached to nanotube surface. We assume that the 
crosslinking is taking place between individual nanotubes and each nanotube behaves as a crosslinker since more than one allylamine monomer linked on the surface of a nanotube structure and because of this reason the polymerization is not chain-polymerization. It creates a network between individual nanotubes because of multiple allylamine molecules on the surface of single nanotubes. The density of created porous 3D-CNTs solid structure is light-weight the density of $0.02 \mathrm{mg} / \mathrm{mm}^{3}$ (Figure 1c-d). The surface modification and crosslinking between individual nanotubes is confirmed by spectroscopic techniques (Figure 2). Raman spectroscopy is one of the most effective way to confirm the chemical modifications on the surface CNTs. Raman spectra of nanotubes in general has two peaks, G-band and D-band [19]. The G-band is related to graphitic carbon and the D-band is related to $\mathrm{sp}^{3}$ vibrations, which comes from the disorders on the surface of nanotubes [20]. The ratio between $D$ and G-band $\left(I_{D}: I_{G}\right)$, which is used to obtain information about structural changes as a result of functionalization, show the defect density of the surface of CNTs. The Raman spectra of these CNTs show D-band and Gband at $1348 \mathrm{~cm}^{-1}$ and $1582 \mathrm{~cm}^{-1}$ respectively (Figure 2a). The intensity ratio between the Dband and G-band $\left(\mathrm{I}_{\mathrm{D}} / \mathrm{I}_{\mathrm{G}}\right)$ increased from 0.6 to 0.93 as a result of surface functionalization and crosslinking $[13,20]$. Fourier transform infrared spectroscopy (FT-IR) results is shown in Figure 2b. The peak at $\sim 1708 \mathrm{~cm}^{-1}$, which appears on oxidized CNTs, functionalized and crosslinked CNTs, shows the $-\mathrm{C}=\mathrm{O}$ functional groups on the surface of CNTs [21]. After functionalization of CNTs with allylamine monomer and free radical polymerization alkyl $\left(-\mathrm{CH}_{2^{-}}\right)$peak at $2940 \mathrm{~cm}^{-}$ 1 and broad amine peak (-NH-) at $\sim 3420 \mathrm{~cm}^{-1}$ showed up (Figure 2b) [22]. XPS characterization was used for quantitative chemical analysis of CNTs before and after surface functionalization as well as the crosslinked samples (CR-CNTs). The core C1s peak is approximately at $285 \mathrm{eV}$ and the peak position of oxygen is around $532 \mathrm{eV}$ (Figure S1a) [23]. After CNTs functionalized with allylamine monomer and polymerized via free radical polymerization, N1s peak appeared around $400 \mathrm{eV}$ (Figure S1b, c). According to XPS characterization, the surface of nanotubes functionalized $\sim 6 \%$ with the allylamine monomer (Figure $\mathrm{S} 1$ ). Figure $2 \mathrm{c}$ is related to pure CNTs, the peak at $284.7 \mathrm{eV}$ and $286.3 \mathrm{eV}$ are related to $\mathrm{C}-\mathrm{C}$ and $\mathrm{O}-\mathrm{C}=\mathrm{O}$ bonds. Figure $2 \mathrm{~d}$ that is $\mathrm{XPS}$ spectrum after polymerization of allylamine functionalized $\mathrm{CNTs}$, shows that $\mathrm{O}-\mathrm{C}=\mathrm{O}$ peaks shifted to 285.3 and C-N peak showed up at 286.5, which indicate that functionalization and polymerization reactions took place $[13,23,24]$. The reason peaks shifted is most probably the conversion of $\mathrm{HO}-\mathrm{C}=\mathrm{O}$ bond to $\mathrm{HN}-\mathrm{C}=\mathrm{O}$ bond after chlorination of $\mathrm{HO}-\mathrm{C}=\mathrm{O}$ and reaction with 
allylamine monomer. Beside spectroscopic characterization, the morphology and microstructure of nanotube based 3D-solid structure were revealed by SEM and TEM.

Figure 2e-f show SEM images of non-crosslinked (n-CNTs) and crosslinked nanotubes (CRCNTs) after freeze-drying process. While $n$-CNTs nanotubes have only van der Waals and $\pi-\pi$ interactions between individual nanotubes, chemically crosslinked nanotubes have covalent bonding in addition to van der Waals and $\pi-\pi$ interactions. Therefore, in Figures 2e-f, the porosity of CNTs after crosslinking is more organized than before crosslinking because covalent crosslinking makes nanotube bundles well-organized. Figure S2 shows high resolution transmission electron microscopy analysis of interconnected 3D-solid network of nanotube structures. Nanotubes are highly crosslinked because allylamine functionalized CNTs behave as monomers and cross-linkers.

The n-CNTs did not form as a solid structure after freeze-drying process. We added $2 \%$ polyvinyl alcohol (PVA) in both CR-CNTs and n-CNTs and used freeze-drying process to create and compare macrostructure of non-crosslinked and crosslinked nanotubes. Using PVA as a reinforcement gives us opportunity to compare crosslinked and non-crosslinked CNTs foam. Compressive mechanical properties of CR-CNTs (0.3349 1/mm 0.3333 1/mm 0.1116 1/.mm²)

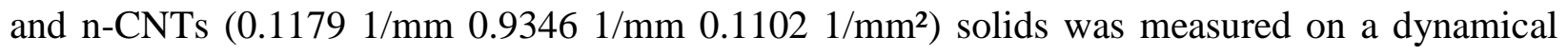
mechanical analysis (DMA) instrument. Figure 3a shows load-unload stress-strain curves. The compression loading curves indicate that the sponges can be compressed down to about $80 \%$ strain. As the load on the material increases, the nature of the curve remains the same during load-unloading test (Figure 3a). The compression stress of crosslinked nanotube foam (0.1MPa) is higher than the stress of non-crosslinked nanotube foam $(0.03 \mathrm{MPa})$ at the same strain $(72 \%)$. It shows that the unloading curve cannot recoil to the primary point, which indicate that the foam has irreversible buckling and the porous starts to deform. The unloading-reloading tests reveal hysteresis, indicative of internal friction and viscoelasticity. The CR-CNTs structure can distribute the load throughout the network to avoid concentrated stress and failure effectively enhance the mechanical strength of the 3D-structure. The stiffness of 3D nanotube foam structures was tested as a function of time at $0.5 \%$ strain (Figure $3 \mathrm{~b}$ ). It shows that the stiffness of 3D nanotube solid architectures increases as the time increase. The initial stiffness of n-CNTs and CR-CNTs is $112 \mathrm{~N} / \mathrm{m}$ and $123 \mathrm{~N} / \mathrm{m}$, respectively. The stiffness of 3D-nCNTs increases to 
$126 \mathrm{~N} / \mathrm{m}$, while crosslinked 3D-CNTs increases to $186 \mathrm{~N} / \mathrm{m}$ in $5 \mathrm{~min}$ and the stiffness of both crosslinked and n-CNT solid structures become constant. Figure 3c shows the storage modulus of nanotube solid structures as a function of frequency. The storage modulus increase with increasing the frequency. As it is shown in the figure $3 \mathrm{c}$, the storage modulus of non-crosslinked nanotubes structures increases $68 \%$ while the modulus of crosslinked nanotubes increases $168 \%$. This improvement can be explained by the covalent interconnection between individual nanotubes. While n-CNTs have physical interactions such as Van der Waals, $\pi-\pi$ and hydrophobic interactions, the CR-CNTs architecture is interconnected via covalent bonding. This covalent bonding shows obviously reinforcement to the CNTs based 3D solid building blocks. The variation of stiffness as a function of force measured at a constant frequency of $1 \mathrm{~Hz}$ (Figure 3d). As it can be seen from Figure 3d, the stiffness increases with the force, probably due to both the entanglements between nanotubes and the load transfer. The stiffness of crosslinked 3D solid CNTs is $192 \mathrm{~N} / \mathrm{m}$, which is higher than that of non-crosslinked CNTs $(132 \mathrm{~N} / \mathrm{m})$ because of covalent interconnections. The load transfer in the crosslinked 3D-CNTs structures is different from that of non-crosslinked solid structures due to the covalent interconnection, which may be one of the factors in the stiffening. The elastic modulus determined from stress-strain curve shows the enhancement of young modulus to $385 \%$ after crosslinking. G. Lalwani and co-worker reported $118 \%$ enhancement of young modulus after crosslinking of nanotubes using benzoyl peroxide [25].

We used fully atomistic reactive molecular dynamic simulations to reveal fracturing mechanism between interconnected and non-crosslinked individual nanotubes into the 3D-CNTs solid. All the shear fracture force computer experiments are computed using classical molecular dynamics (MD) with its numerical implementation in the large-scale atomic/molecular massively parallel simulator [26] (LAMMPS), using: 1) a combination of Reax Force Field (ReaxFF) parameterizations for fullerene [27] and silicon carbide [28], 2) a time step of 0.25 femtoseconds, the 3) Nosé-Hoover thermostat at room temperature, and 4) the employment of a zigzag $(9,0)$ carbon nanotube (CNT) on account of its greater bonding capability than the armchair analogue [29].

As explained by T. Tsafack et al.[30], the shear fracture force experiment is designed to measure the stability and/or strength of a given structure as well as the strength of the bonding 
scheme thereof. In configurations I, II, III, IV, and V depicted in Figure 4, two tubes with different orientations are connected through the polymer chain $\mathrm{XL}=\left\{-\mathrm{CO}-\mathrm{NH}-\mathrm{CH}_{2}-\mathrm{CH}_{-}-\mathrm{CH}_{2}-\right\}$ and kept vibrating around their equilibrium positions thanks to two handles located at the end of both tubes in all configurations leaving CNT-XL interfaces free to interact. An initial equilibration process (NVT) takes place for 25 picoseconds involving the whole structure (minus the handles) followed by another equilibration process (NVT) which occurs in continuing cycles of 1 picosecond each with the right handle (configurations I, II, III, IV) or bottom handle (configuration V) staying fixed and the left handle (configurations I, II, III, IV) or top handle (configuration V) moves down (configurations I, II, III, IV) or up (configuration V) by $0.05 \AA$. The force on the tube moving tube, i.e., left tube in configurations I, II, III, IV and top tube in configuration $\mathrm{V}$, is calculated before the beginning of the next cycle until XL polymer chain breaks. The force at the breaking point $-\mathrm{CH}_{2}-\mathrm{CH}_{2}$ ) is then recorded as the fracture force (see supplemental videos).

Three-dimensional configurations CNT, CNT-COOH, and CNT-XL respectively with no crosslinking, $15 \% \mathrm{COOH}$ functional groups and $\mathrm{XL}$ as crosslinkers connecting three randomly oriented tubes are: 1) equilibrated (NVT) for 7.5 picoseconds, 2) compressed by $\sim 30 \%$ in all three dimensions for 7.5 picoseconds, and 3) put into an NPT ensemble for 15 picoseconds to allow the box to relax into a more stable configuration. The tubes in the resulting boxes are kept fixed through handles located at both ends of the tubes and as the whole structure is being equilibrated, the handles in the top most tube are pulled upward by $0.05 \AA$ every picosecond while the force on the tube is being calculated.

The fracture force experiment in Figure $4 \mathrm{~b}$ shows a significant strength difference (1.5 $\mathrm{nN}$ ) between the strongest (configuration V) and the weakest (configuration III) configurations, configurations II and IV being almost equivalently strong and both stronger than configuration I. The aforementioned performance difference among configurations I, II, III, IV and V is to be ascribed to the reactivity of interfacial ends and the orientation of the non-moving tube. In fact, both reactive $\mathrm{sp}^{2}$ interfacial ends in configuration $\mathrm{V}$ are responsible for the structure being the strongest one, the reactive $\mathrm{sp}^{2}$ interfacial end on the right tube of configuration IV is similarly responsible for its strength. Configuration II gets its strength from the orientation of the right (nonmoving) tube vibrating partly in the opposite direction to partly in the direction of the left 
(moving) tube. Conversely, configurations III and I appear to be the weakest because of the weaker $\mathrm{sp}^{3}$ interfacial bonding and the orientation of the non-moving tube non favoring the structures' stability.

The randomly oriented three-dimensional structures in Figure 4c show (Figure 4d) the CNT-XL structure being roughly twice as strong as the pristine $\mathrm{CNT}$ and the CNT-COOH structures. The Van der Waals repulsive interaction between pristine CNTs confirming their hydrophobic nature (CNT in Figure 4c-d) and the weak CNT-COOH-COOH-CNT (CNT-COOH in Figures 4c-d) appear to be weaker than the CNT-XL-XL-CNT configuration as evidenced by experiments (Figures 3a-d).

The surface area and porosity of the 3D porous crosslinked and non-crosslinked CNT solids were conducted via full nitrogen adsorption/desorption isotherms as shown in Figure 5. The curves reveal that both crosslinked and non-crosslinked nanotube solids possess type II adsorption/desorption isotherms, corresponding to macroporous structure with relatively weak interactions, which also exhibits a negligible concave section (Figure 5a) [31]. The surface area of nanotubes is affected by several factors such as number of walls, diameter of nanotubes, impurities, surface functionalization etc. One of the most important factors, which affect the surface area of bulk nanotubes is the space between individual nanotubes. As grown nanotubes bundle/powder stay together by hydrophobic, $\pi-\pi$, van der Waals etc. interactions. As shown in Figure 1, compared to chemically interconnected nanotubes by a linker, the distance between nanotubes, which stay together by physical interactions is smaller [14]. The BET surface area $\left(\mathrm{S}_{\mathrm{BET}}\right)$ of $\mathrm{n}$-CNTs solid is $223 \mathrm{~m}^{2} / \mathrm{g}$. The surface area has increased to $374 \mathrm{~m}^{2} / \mathrm{g}$ after chemical crosslinking because the chemical crosslinking generates expansion between individual nanotubes, thus the surface area of nanotubes bundle increase compared to non-crosslinked bundle [14]. Additionally, the chemical interconnection prevents nanotubes to aggregate, which decrease the volume/distance between individual nanotubes and favors the creation of a more ordered porous structures [13]. DFT method for pore size distribution and cumulative pore volume of crosslinked and non-crosslinked nanotube solids is shown in Figures $4 \mathrm{~b}$ and $4 \mathrm{c}$. The pore size and the pore volume increase after crosslinking. The cumulative pore volume of crosslinked and pristine nanotube solids is $0.27 \mathrm{~cm}^{3} / \mathrm{g}$ and $0.18 \mathrm{~cm}^{3} / \mathrm{g}$ respectively (Figure $4 \mathrm{~b}$ ). 
Such 3D porous nanotube solids have many interesting properties such as higher surface area than original CNTs, thermal and chemical stability, and hydrophobic surface properties. Therefore, they have a broad range of applications ranging from catalyst supports [32] and supercapacitors [33] to adsorbents [13] and gas storage [14]. One of the crucial applications of the light-weight, porous materials with high surface area is the adsorption of $\mathrm{CO}_{2}$ that is the process in which $\mathrm{CO}_{2}$ molecules adhere to a surface of nanotubes[34-36]. $\mathrm{CO}_{2}$ emission is one of the major reason of global warming because of the fossil fuels. Recently, a huge attention has been given to develop new low-cost and effective methods for capturing $\mathrm{CO}_{2}$ to reduce the emission in the atmosphere. Different type of materials has been used toward the aim of the $\mathrm{CO}_{2}$ adsorption such as aqueous amine, metal-organic frameworks [37], zeolites [38], mesoporous materials [39] etc. The current $\mathrm{CO}_{2}$ adsorption technologies have some disadvantages such as high-cost, corrosion in the equipment and high-energy consumption. Hence, the development of novel materials and new technologies for $\mathrm{CO}_{2}$ adsorption and storage is needed. Surface area, pore volume and functional groups on the surface of the materials are some effective factors to improve the $\mathrm{CO}_{2}$ adsorption capability of the material. Although, the surface area of CNTs are lower than most of the porous carbon materials commonly used for $\mathrm{CO}_{2}$ capture, such as activated carbons[40,41], it has been shown that with chemical modifications, particularly with 3-aminopropyltriethoxysilane, can improve physicochemical properties, thus enhancing $\mathrm{CO}_{2}$ sorption capacity [42-45]. Here, we introduce a method, particularly to increase the surface area and porosity of CNTs using chemical functionalization followed by crosslinking. Moreover, the crosslinking agent containing amide functional groups that enhances the selectivity of $\mathrm{CO}_{2}$ capture [40]. Thus, we have obtained more efficient material as a $\mathrm{CO}_{2}$ sorbent.

Among the different strategies for reducing $\mathrm{CO}_{2}$, much effort has been devoted to its capture and storage. Here, we demonstrate 3D-CNTs building blocks to capture and store of $\mathrm{CO}_{2}$ (Figures 5d, S5 and S6). While the non-crosslinked nanotubes have captured $136 \mathrm{mg} / \mathrm{g} \mathrm{CO}_{2}$, the crosslinked 3D-nanotube solids have captured $198 \mathrm{mg} / \mathrm{g}$ of $\mathrm{CO}_{2}$ at 54 bars because of the improved surface area, as a result of highly ordered porosity. The isosteric heat of absorption (Qst) of $\mathrm{CO}_{2}$ on the surfaces of CNTs and 3D-CNTs were calculated using low pressure $\mathrm{CO}_{2}$ sorption isotherms at $25^{\circ} \mathrm{C}$ and $40{ }^{\circ} \mathrm{C}$ as shown in Figure S5 and S6. The Qst values for CNTs is $\sim 17 \mathrm{~kJ} / \mathrm{mol}$ and quite comparable with the values for undoped carbon materials [46-49]. However, Qst value for 3D-CNTs starts from $\sim 60 \mathrm{~kJ} / \mathrm{mol}$ and drops to $\sim 17 \mathrm{~kJ} / \mathrm{mol}$ at higher 
pressures upon filling the macropores with $\mathrm{CO}_{2}$ molecules. The high Qst values for 3D-CNTs is indicative of weakly chemisorbed nature of $\mathrm{CO}_{2}$ molecules on nitrogen functioinal groups within the pores of 3D-CNTs [40].

\section{CONCLUSION}

In conclusion, we reported chemically crosslinked light-weight and porous 3D carbon nanotube solid structures. As a result of chemical crosslinking mechanical properties of the crosslinked solid structures improved compared to non-crosslinked nanotubes. In addition to the improved mechanical properties, surface area and porosity increased as well. Fracturing mechanism of chemically interconnected nanotubes and physically integrated nanotubes have been investigated by fully atomistic reactive $\mathrm{MD}$ simulations. The $\mathrm{CO}_{2}$ adsorption improves by $46 \%$ after crosslinking at 54 bars due to the increased surface area, nitrogen functional groups and porosity of the 3D-CNTs solids.

Acknowledgements. This work has been supported by U.S. Department of Defense: U.S. Air Force Office of Scientific Research (AFOSR) for the project "MURI: Synthesis and Characterization of 3-D Carbon Nanotube Solid Networks" through the award \# FA9550-12-10035 and Air Force Office of Scientific Research (AFOSR) for the project "Understanding SelfStrengthening Mechanisms in Nanocomposites" through Grant award \#FA9550-13-1-0084. S.O. and A.D.M gratefully acknowledge the support of the U.S. Department of Energy through the LANL/LDRD Program for this work. S.O. acknowledges financial support from a LANL Director's Postdoctoral Fellowship.

\section{AUTHOR INFORMATION}

\section{Corresponding Author}

E-Mail: ajayan@rice.edu (P. M. Ajayan)

E-Mail: sozden@lanl.gov (S. Ozden)

\section{References}

[1] X.C. Gui, Z.P. Zeng, A.Y. Cao, Z.Q. Lin, H.Q. Zeng, R. Xiang, et al., Elastic shape recovery of carbon nanotube sponges in liquid oil, J. Mater. Chem.. 22 (2012) 18300-18305. doi:Doi 10.1039/C2jm33686c. 
[2] Q. Peng, Y. Li, X. He, X. Gui, Y. Shang, C. Wang, et al., Graphene nanoribbon aerogels unzipped from carbon nanotube sponges, Adv. Mater.. 26 (2014) 3241-3247. doi:10.1002/adma.201305274.

[3] S. Vinod, C.S. Tiwary, P.A. da Silva Autreto, J. Taha-Tijerina, S. Ozden, A.C. Chipara, et al., Low-density three-dimensional foam using self-reinforced hybrid two-dimensional atomic layers., $\quad$ Nat. Commun.. 5 (2014) 4541. doi:10.1038/ncomms5541.

[4] J.M. Romo-Herrera, M. Terrones, H. Terrones, S. Dag, V. Meunier, Covalent 2D and 3D networks from 1D nanostructures: Designing new materials, Nano Lett. 7 (2007) 570-576. doi:10.1021/nl0622202.

[5] L. Ma, A.H.C. Hart, S. Ozden, R. Vajtai, P.M. Ajayan, Advances of carbon nanomaterials, Faraday Discuss. 173 (2014) 9-46. doi:10.1039/C4FD90039A.

[6] S. Ozden, C.S. Tiwary, A.H.C. Hart, A.C. Chipara, R. Romero-Aburto, M.-T.F. Rodrigues, et al., Density variant carbon nanotube interconnected solids., Adv. Mater.. 11 (2015) 1842-1850. doi:10.1002/adma.201404995.

[7] S. Ozden, G. Brunetto, N.S. Karthiselva, D.S. Galvão, A. Roy, S.R. Bakshi, et al., Controlled 3D Carbon Nanotube Structures by Plasma Welding, Adv. Mater. Inter. 3 (2016) 1-8. doi:10.1002/admi.201500755.

[8] D. Wei, Y. Liu, The intramolecular junctions of carbon nanotubes, Adv. Mater.. 20 (2008) 2815-2841. doi:10.1002/adma.200800589. 
[9] C. Shan, W. Zhao, X.L. Lu, D.J. O’Brien, Y. Li, Z. Cao, et al., Three-dimensional nitrogen-doped multiwall carbon nanotube sponges with tunable properties, Nano Lett. 13 (2013) 5514-5520. doi:10.1021/nl403109g.

[10] S. Ozden, C.S. Tiwary, A.H.C. Hart, A.C. Chipara, R. Romero-Aburto, M.-T.F. Rodrigues, et al., Density Variant Carbon Nanotube Interconnected Solids., Adv. Mater. 27 (2015) 1-9. doi:10.1002/adma.201404995.

[11] D.P. Hashim, N.T. Narayanan, J.M. Romo-Herrera, D.A. Cullen, M.G. Hahm, P. Lezzi, et al., Covalently bonded three-dimensional carbon nanotube solids via boron induced nanojunctions., Scientific Reports. 2 (2012) 1-8. doi:10.1038/srep00363.

[12] L. Camilli, C. Pisani, E. Gautron, M. Scarselli, P. Castrucci, F. D’Orazio, et al., A threedimensional carbon nanotube network for water treatment., Nanotechnology. 25 (2014) 65701. doi:10.1088/0957-4484/25/6/065701.

[13] S. Ozden, T.N. Narayanan, C.S. Tiwary, P. Dong, A.H.C. Hart, R. Vajtai, et al., 3D macroporous solids from chemically cross-linked carbon nanotubes, Small. 11 (2015) 688-693. doi:10.1002/smll.201402127.

[14] A.D. Leonard, J.L. Hudson, H. Fan, R. Booker, L.J. Simpson, K.J. O’Neill, et al., Nanoengineered carbon scaffolds for hydrogen storage., J. Am. Chem. Soc. 131 (2009) 723-8. doi:10.1021/ja806633p.

[15] M. Terrones, F. Banhart, N. Grobert, J.-C. Charlier, H. Terrones, P.M. Ajayan, Molecular Junctions by Joining Single-Walled Carbon Nanotubes, Phys. Rev. Lett.. 89 (2002) 75505. doi:10.1103/PhysRevLett.89.075505. 
[16] D. R. Rolison, J. W. Long, J. C. Lytle, A. E. Fischer, C.P. Rhodes, T.M. Mcevoy, E. Bourg, A.M. Lubers, Multifunctional 3D nanoarchitectures for energy storage and conversion.Chem. Soc. Rev. 38 (2009) 226-252. doi:10.1039/b801151f.

[17] J. Zhu, H. Peng, F. Rodriguez-Macias, J.L. Margrave, V.N. Khabashesku, A.M. Imam, et al., Reinforcing epoxy polymer composites through covalent integration of functionalized nanotubes, Adv. Func. Mater. 14 (2004) 643-648. doi:10.1002/adfm.200305162.

[18] Ş. Özden, S.Ü. Çelik, A. Bozkurt, Synthesis and proton conductivity studies of polystyrene-based triazole functional polymer membranes, J. Polym. Sci., Part A: Polym. Chem. 48 (2010) 4974-4980. doi:10.1002/pola.24293.

[19] M.S. Dresselhaus, G. Dresselhaus, R. Saito, A. Jorio, Raman spectroscopy of carbon nanotubes, Phys. Rep . 409 (2005) 47-99. doi:10.1016/j.physrep.2004.10.006.

[20] W. Kiefer, Raman spectroscopy of covalently functionalized single-wall carbon nanotubes, J. Raman Spectrosc. 38 (2007) 1538-1553. doi:10.1002/jrs.

[21] J. Chen, M. A. Hamon, H. Hu, Y. Chen, A.M. Rao, P.C. Eklund, R.C. Haddon, Solution properties of single-walled carbon nanotubes, Science 282 (1998) 0-4. DOI: 10.1126/science.282.5386.95.

[22] S.Ü. Ozden, Sehmus Çelik, A. Bozkurt, Synthesis and proton conductivity studies of azole functional organic electrolytes, Electrochim. Acta. 18 (2010) 101-107. doi:10.1007/s11581-011-0600-0. 
[23] T.I.T. Okpalugo, P. Papakonstantinou, H. Murphy, J. McLaughlin, N.M.D. Brown, High resolution XPS characterization of chemical functionalised MWCNTs and SWCNTs, Carbon. 43 (2005) 153-161. doi:10.1016/j.carbon.2004.08.033.

[24] P. Ayala, A. Gru, T. Gemming, D. Grimm, C. Kramberger, M.H. Ru, et al., Tailoring Ndoped single and double wall carbon nanotubes from a nondiluted carbon / nitrogen feedstock J. Phys. Chem. C. 111 (2007) 2879-2884. DOI: 10.1021/jp0658288

[25] G. Lalwani, A.T. Kwaczala, S. Kanakia, S.C. Patel, S. Judex, B. Sitharaman, Fabrication and characterization of three-dimensional macroscopic all-carbon scaffolds, Carbon. 53 (2013) 90-100. doi:10.1016/j.carbon.2012.10.035.

[26] S. Plimpton, Fast parallel algorithms for short-range molecular dynamics, J. Comput. Phys. 117 (1995) 1-19. doi:10.1006/jcph.1995.1039.

[27] R. Paupitz, C.E. Junkermeier, A.C.T. Van Duin, P.S. Branicio, Fullerenes generated from porous structures, Phys. Chem. Chem. Phys.. $16 \quad$ (2014) 25515-25522. doi:10.1039/C4CP03529A.

[28] D.A. Newsome, D. Sengupta, H. Foroutan, M.F. Russo, A.C.T. Van Duin, Oxidation of silicon carbide by $\mathrm{O}_{2}$ and $\mathrm{H}_{2} \mathrm{O}$ : A ReaxFF reactive molecular dynamics study, J. Phys. Chem. C. 116 (2012) 16111-16121. DOI: 10.1021/jp306391p.

[29] M.G. Ahangari, A. Fereidoon, M. Jahanshahi, M.D. Ganji, Electronic and mechanical properties of single-walled carbon nanotubes interacting with epoxy: A DFT study, Physica E. 48 (2013) 148-156. doi:10.1016/j.physe.2012.12.013. 
[30] T. Tsafack, J.M. Alred, K.E. Wise, B. Jensen, E. Siochi, B.I. Yakobson, Exploring the interface between single-walled carbon nanotubes and epoxy resin, Carbon. 105 (2016) 600-606. doi:10.1016/j.carbon.2016.04.066.

[31] I.A.A.C. Esteves, F.J.A.L. Cruz, E.A. Mu, S. Agnihotri, Determination of the surface area and porosity of carbon nanotube bundles from a Langmuirian analysis of sub- and supercritical adsorption data. Carbon 7 (2010) 2-10. doi:10.1016/j.carbon.2008.11.044.

[32] Y. Yan, J. Miao,a Z. Yang, F. Xiao, H. B. Yang, B. Liu, Y. Yang Carbon nanotube catalysts : recent advances in synthesis, characterization and applications, Chem. Soc. Rev. 44 (2015) 3295-3346. doi:10.1039/C4CS00492B.

[33] Y. Rangom, X.S. Tang, L.F. Nazar, Carbon nanotube-based supercapacitors with excellent ac line filtering and rate capability via improved interfacial impedance, ACS Nano. 9 (2015) 7248-7255. DOI: 10.1021/acsnano.5b02075.

[34] Y. Bae, R.Q. Snurr, Development and evaluation of porous materials for carbon dioxide separation and capture, Angew. Chem. 50 (2011) 11586-11596. doi:10.1002/anie.201101891.

[35]M.E. Casco, M. Mart1, F. Rodr1, Effect of the porous structure in carbon materials for $\mathrm{CO}_{2}$ capture at atmospheric and high-pressure, Carbon, 7 (2013) 3-8. doi:10.1016/j.carbon.2013.09.086.

[36] D.M.D. Alessandro, B. Smit, J.R. Long, Carbon Dioxide Capture Carbon Dioxide Capture: Prospects for New Materials. Angew. Chem. , 49 (2010) 6058-6082. doi:10.1002/anie.201000431.

[37] K. Zhang, Z. Qiao, J. Jiang, Molecular design of zirconium tetrazolate metal-organic 
frameworks for $\mathrm{CO}_{2}$ Capture. Cryst. Growth Des. $17 \quad$ (2017) 543-549. doi: 10.1021/acs.cgd.6b0140.

[38] T.H. Nguyen, H. Gong, S.S. Lee, T-H. Bae. Amine-appended hierarchical Ca-A zeolite for enhancing $\mathrm{CO}_{2} / \mathrm{CH}_{4}$ selectivity of mixed-matrix membranes. ChemPhysChem. 17 (2016) 31653169. doi: $10.1002 / \mathrm{cphc} .201600561$

[39] K.S.Lakhi, A.V. Baskar, J.S.M. Zaidi, S.S. Al-Deyab, M. El-Newehy, J-H. Choy J-H, et al. Morphological control of mesoporous $\mathrm{CN}$ based hybrid materials and their excellent $\mathrm{CO}_{2}$ adsorption capacity. RSC Adv. 5 (2015) 40183-40192.

[40] A.S. Jalilov, G. Ruan, C. Hwang, D.E. Schipper, J.J. Tour, Y. Li, et al., Asphalt-derived high surface area activated porous carbons for carbon dioxide capture. Carbon, 7 (2015) 13761382. doi:10.1021/am508858x.

[41] A. E. Creamer, B. Gao. Carbon-Based Adsorbents for Postcombustion $\mathrm{CO}_{2}$ Capture: A Critical Review. Environ. Sci. Technol. 50 (2016) 7276-7289. doi: 10.1021/acs.est.6b00627 [42] C. Lu, H. Bai, B. Wu, F. Su, J. F. Hwang. Comparative Study of $\mathrm{CO}_{2}$ Capture by Carbon Nanotubes, Activated Carbons, and Zeolites. Energy \& Fuels, 22 (2008) 3050-3056.

[43] F. Su, C. Lu, W. Cnen, H. Bai, J. F. Hwang. Capture of $\mathrm{CO}_{2}$ from flue gas via multiwalled carbon nanotubes. Science of the Total Environment 407 (2009) 3017-3023. doi:10.1016/j.scitotenv.2009.01.007

[44] F. Su, C. Lu, A. J. Chung, C. H. Liao. $\mathrm{CO}_{2}$ capture with amine-loaded carbon nanotubes via a dual-column temperature/vacuum swing adsorption. Applied Energy, 113 (2014) 706-712. http://dx.doi.org/10.1016/j.apenergy.2013.08.001 
[45] S.K. Smart, A.I. Cassady, G.Q. Lu, D.J. Martin, The biocompatibility of carbon nanotubes. Carbon 44 (2006) 1034-1047.

[46] D. G. A. Smith, K. Patkowski. Benchmarking the $\mathrm{CO}_{2}$ Adsorption Energy on Carbon Nanotubes. J. Phys. Chem. C 119 (2015) 4934-4948. doi: 10.1021/jp512926n

[47] M. Cinke, J. Li, C.W. Bauschlicher Jr, A. Ricca, M. Meyyappan. $\mathrm{CO}_{2}$ adsorption in singlewalled carbon nanotubes. Chemical Physics Letters 376 (2003) 761-766.

[48]. C. Hsu, C. Lu , F. Su, W. Zeng, W. Chen. Thermodynamics and regeneration studies of $\mathrm{CO}_{2}$ adsorption on multiwalled carbon nanotubes. Chemical Engineering Science 65 (2010) 1354-1361. doi:10.1016/j.ces.2009.10.005

[49] D. J. Babu, M. Lange, G. Cherkashinin, A. Issanin, R. Staudt, J. J. Schneider. Gas adsorption studies of $\mathrm{CO} 2$ and $\mathrm{N} 2$ in spatially aligned double-walled carbon nanotube arrays. Carbon 61 (2013) 616-623 doi: 10.1016/j.carbon.2013.05.045 


\section{Chemically Interconnected Light-Weight 3D-Carbon Nanotube Solid Network for $\mathrm{CO}_{2}$ Uptake}

Sehmus Ozden ${ }^{1 *}$, Thierry Tsafack ${ }^{2}$, Peter S. Owuor ${ }^{2}$, Yilun $\mathrm{Li}^{3}$, Almaz S. Jalilov $^{4}$, Robert

Vajtai $^{2}$, Chandra Sekhar Tiwary ${ }^{2}$, Jun Lou ${ }^{2}$, James M. Tour ${ }^{3}$, Aditya D. Mohite ${ }^{1}$, Pulickel M.

$$
\text { Ajayan }^{1 *}
$$

${ }^{1}$ Materials Physics and Applications Division, Los Alamos National Laboratory, Los Alamos NM, 87545, USA

${ }^{2}$ Department of Materials Science and NanoEngineering, Rice University, Houston, Texas 77005, USA

${ }^{3}$ Department of Chemistry, Rice University, Houston, Texas 77005, USA

${ }^{4}$ Department of Chemistry, King Fahd University of Petroleum and Minerals, Dhahran 31261, Saudi Arabia

(a)
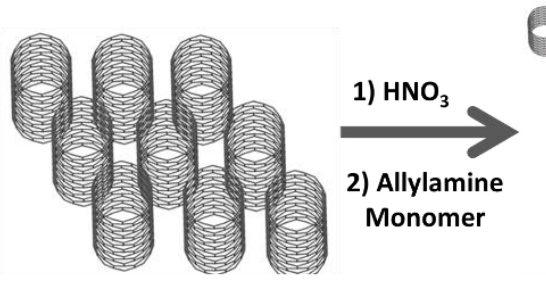

Pristine Nanotube Powder

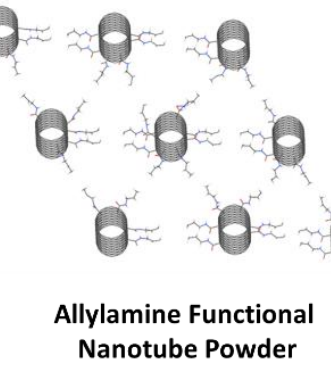

(b)

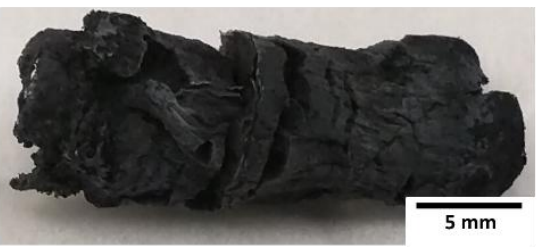

(c)
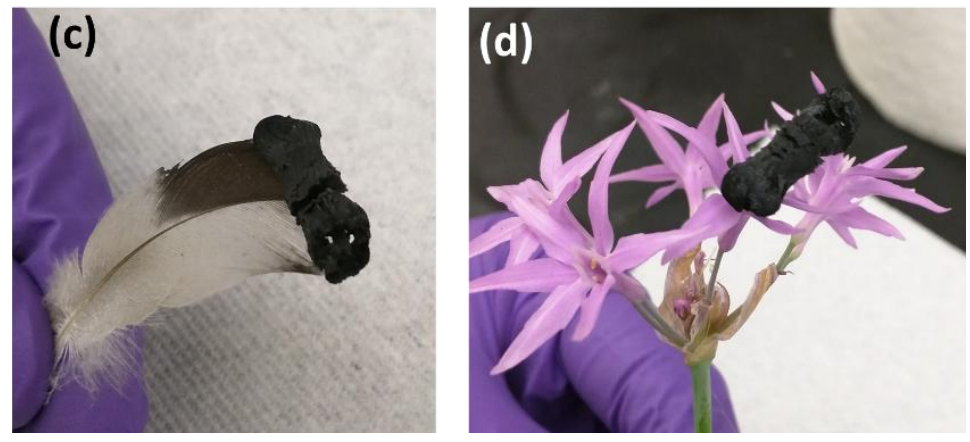
Figure 1. (a) Schematic procedure of 3D-CNTs porous solids synthesis: CNTs functionalized with allylamine monomer followed by free-radical polymerization using AIBN initiator, (b-d) the image of light-weight porous 3D-CNTs foam $\left(0.02 \mathrm{mg} / \mathrm{mm}^{3}\right)$ obtained after freeze-drying process.
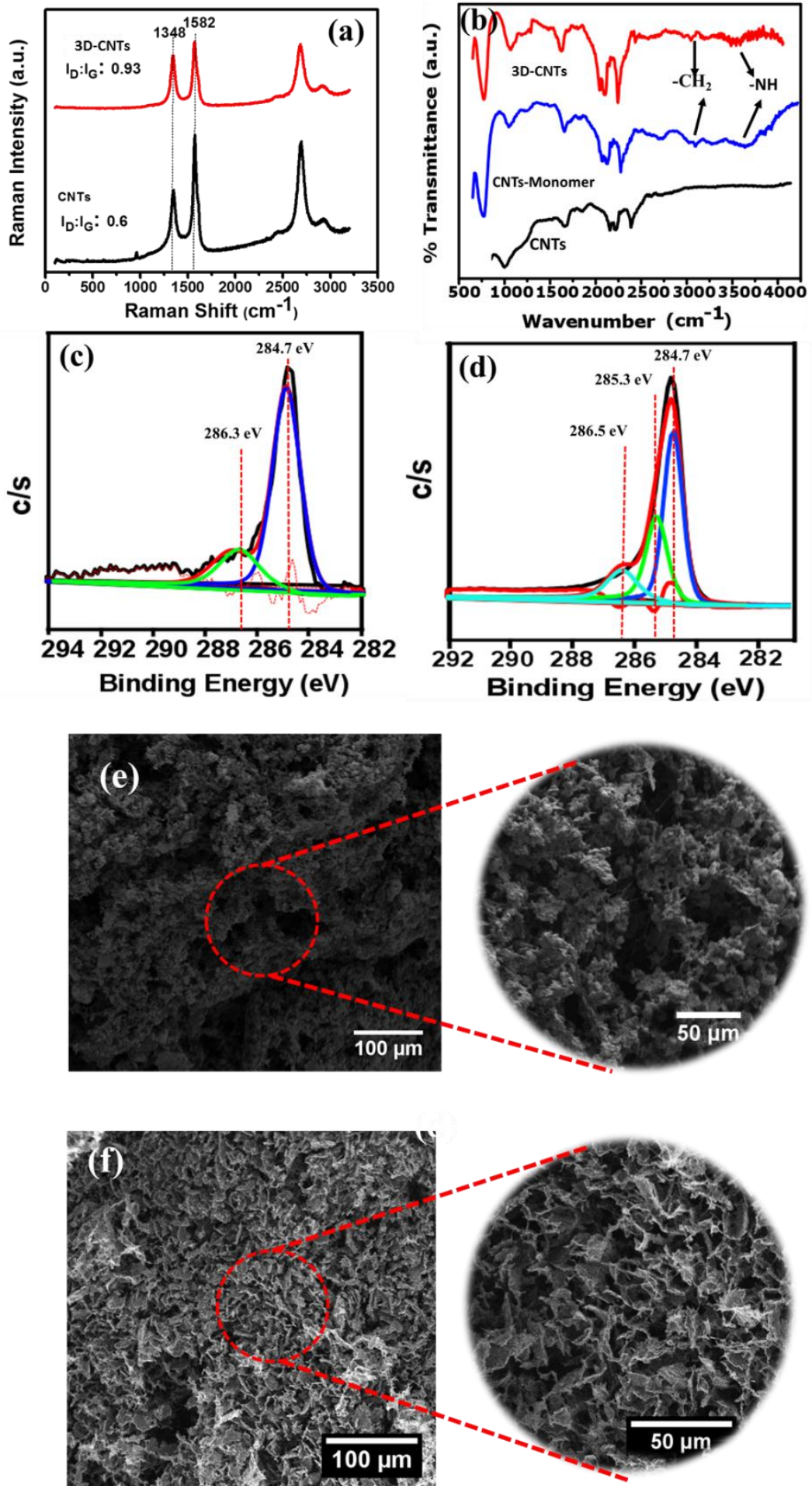

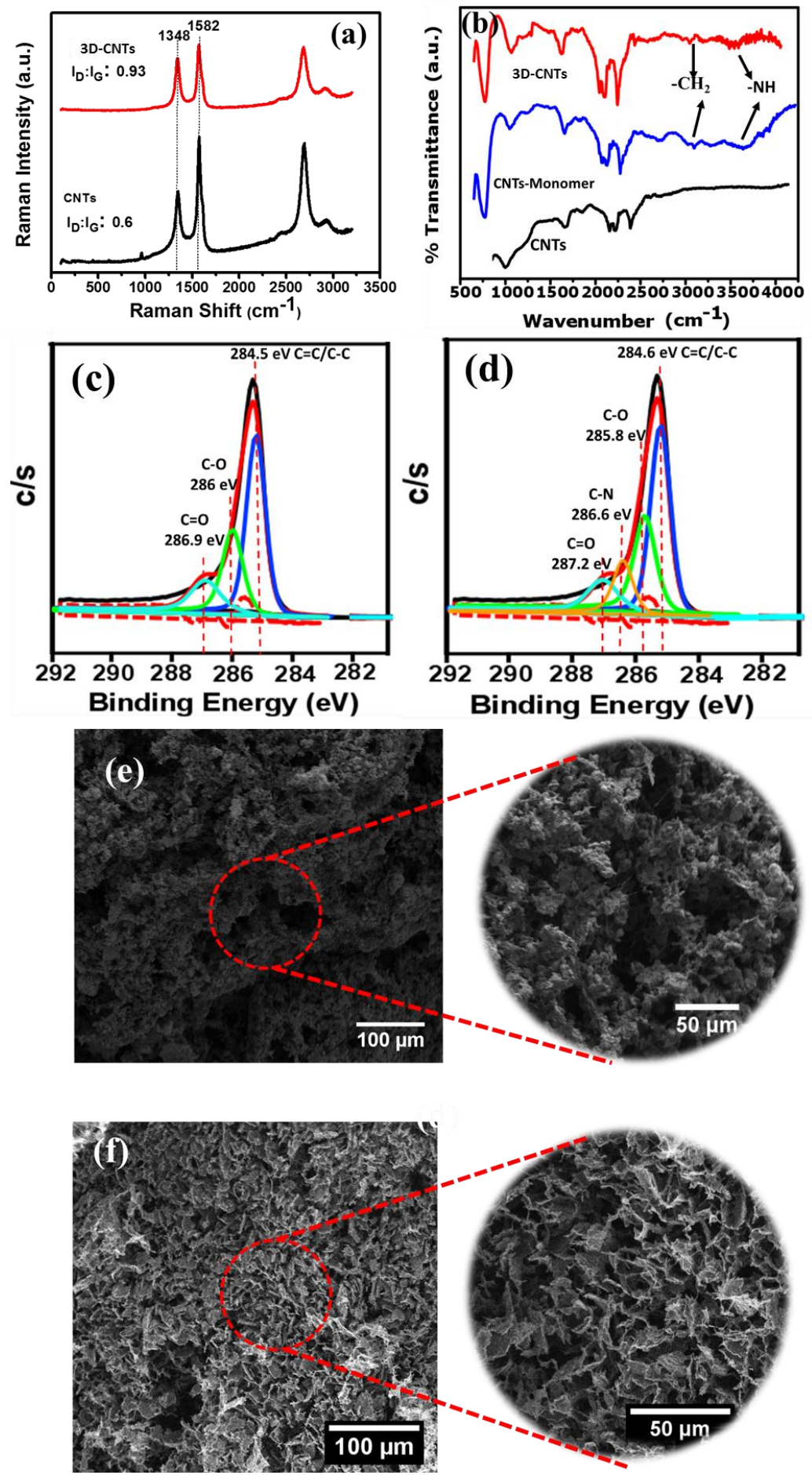

Figure 2. (a) Raman spectra of crosslinked 3D-CNTs solids and non-crosslinked CNTs, (b) FT-IR spectra of oxidized CNTs, monomer functionalized and crosslinked CNTs structures, (c) XPS spectra of pristine CNTs powder, (d) XPS spectra of crosslinked 3D-CNTs solids, (e) SEM image of non-crosslinked CNTs, (f) SEM images of crosslinked 3D-CNTs after freeze-drying process. 
(a)

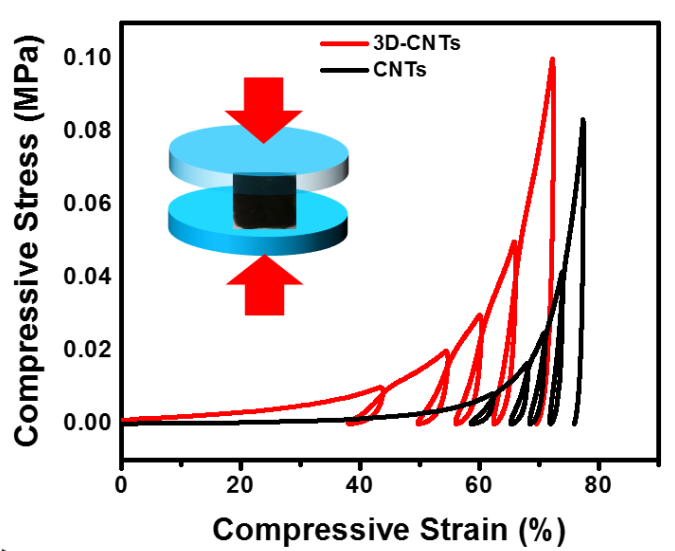

(c)

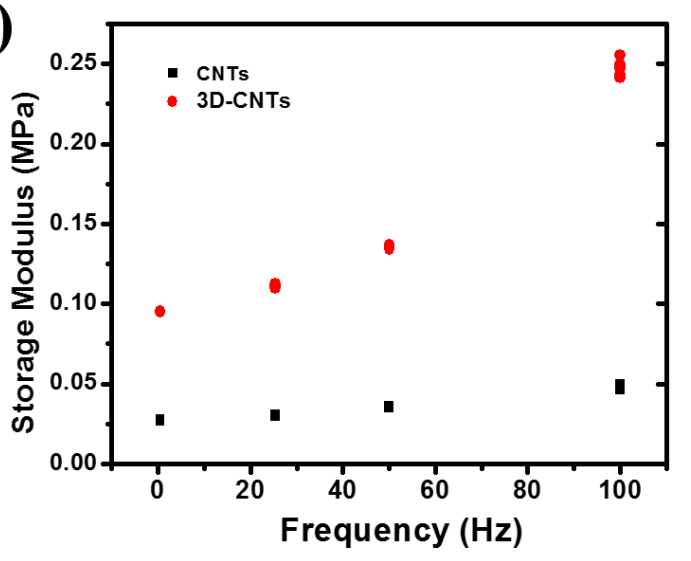

(b)



(d)

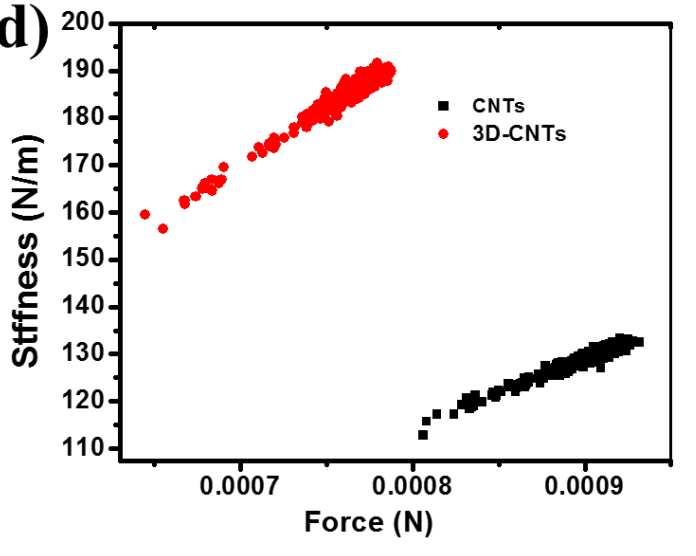

Figure 3. Mechanical characterization of crosslinked 3D-CNTs solids and non-crosslinked CNTs with addition of $2 \%$ PVA for getting foam of non-crosslinked CNTs (a) compressive stress-strain curve, (b) stiffness as a function of time, (c) storage modulus as a function of frequency, (d) Stiffness as a function of force. 
(a)



(c)

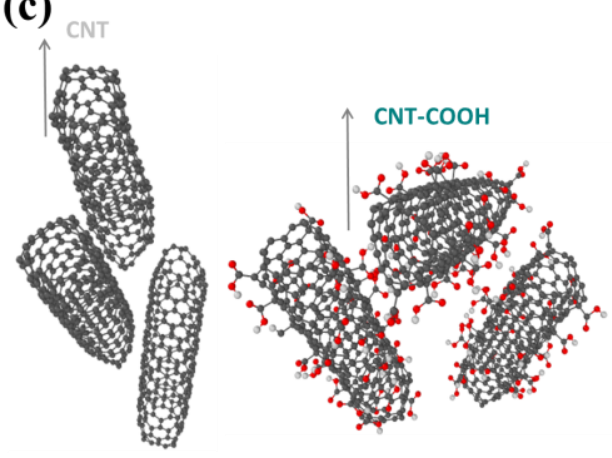

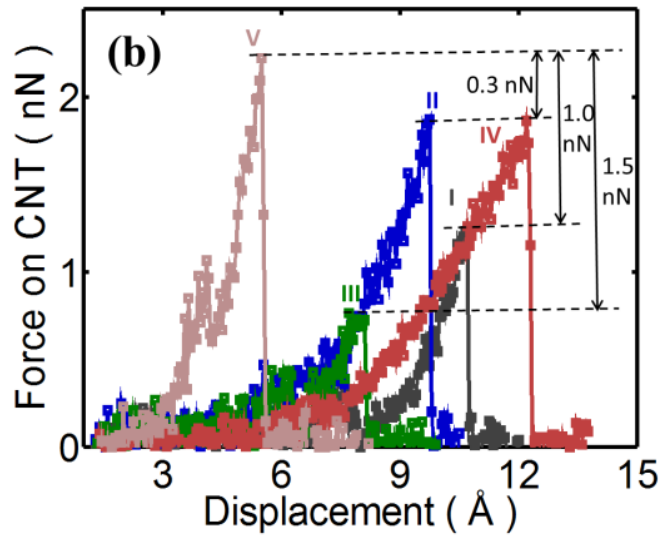

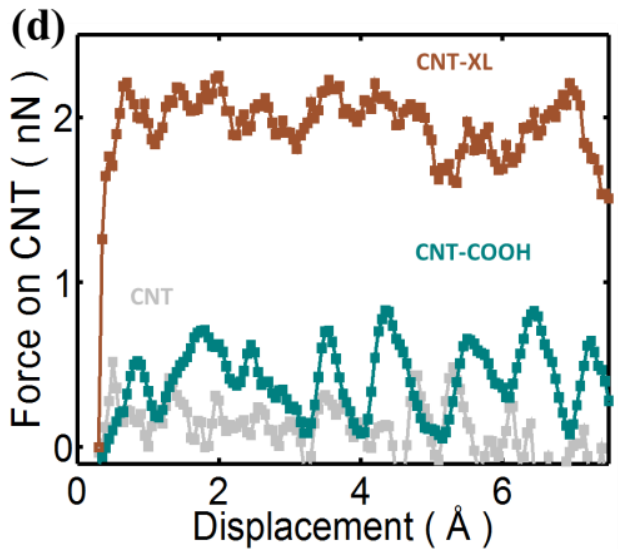

Figure 4. (a) I, II, III, IV, and V CNT-CO-NH-CH2-CH-CH2-CH2-CH-CH2-NH-CO-CNT orientations with left CNT pulling in the directions indicated by the arrow until - $\mathrm{CH} 2-\mathrm{CH} 2$ - bond breaks. (b) Shear force computer experiment showing fracture force on moving CNT for the above configurations, configuration $\mathrm{V}$ having the strongest - $\mathrm{CH} 2-\mathrm{CH} 2$ - bond, (c) Pullout computer experiment in which the (d) CNT (in CNT-XL, CNT-COOH and pristine CNT configurations) is pulled upward while the force on the top CNT is calculated. XL stands for $\mathrm{CO}-\mathrm{NH}-\mathrm{CH} 2-\mathrm{CH}-\mathrm{CH} 2$, (d) An average of 3 covalent crosslinks are created among the 3 CNTs in the CNT-XL configuration whereas no crosslinks are created in the other 2 configurations. 



Figure 5. a) The $\mathbf{N}_{2}$ absorption isotherm of non-crosslinked CNTs and crosslinked 3D-CNT solids (b) Cumulative pore volume as a function of pore size, (c) DFT method pore size distribution of noncrosslinked CNTs and crosslinked 3D-CNT solids, (d) $\mathrm{CO}_{2}$ uptake of non-crosslinked CNTs and crosslinked 3D-CNT solids. 
ToC figure.

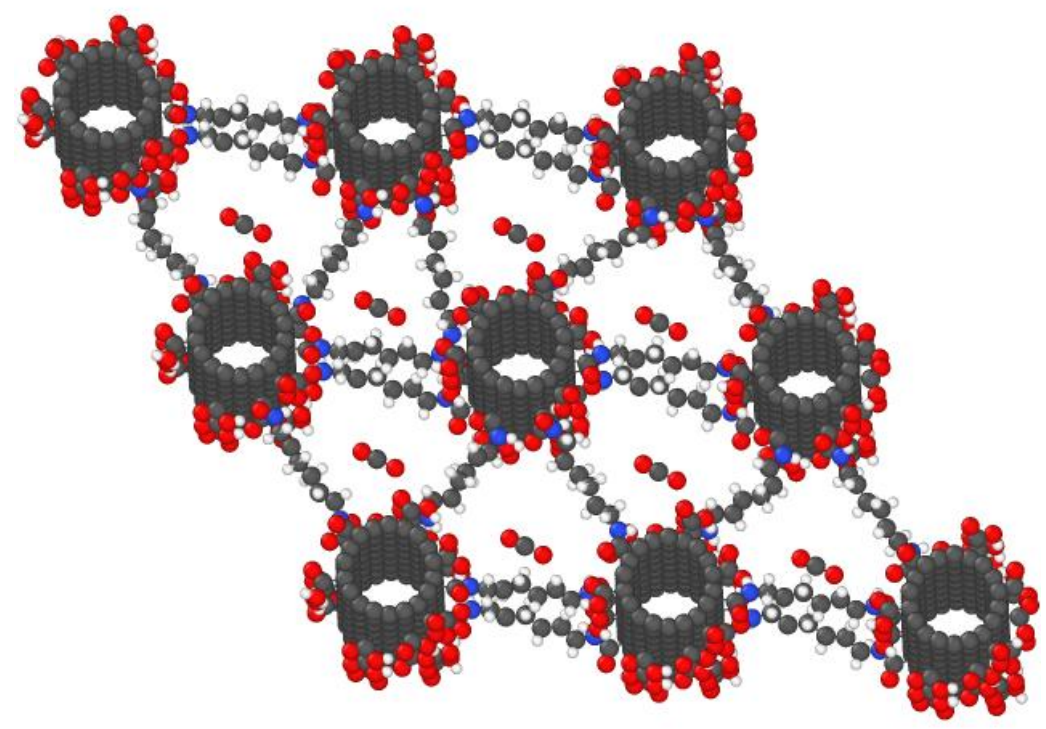

